\title{
Multiuser Relaying over Mixed RF/FSO Links
}

\author{
Nikolaos I. Miridakis, Michail Matthaiou, Senior Member, IEEE, and George K. Karagiannidis, Fellow, IEEE
}

\begin{abstract}
A multiuser dual-hop relaying system over mixed radio frequency/free-space optical (RF/FSO) links is investigated. Specifically, the system consists of $m$ single-antenna sources, a relay node equipped with $n \geq m$ receive antennas and a single photo-aperture transmitter, and one destination equipped with a single photo-detector. RF links are used for the simultaneous data transmission from multiple sources to the relay. The relay operates under the decode-and-forward protocol and utilizes the popular V-BLAST technique by successively decoding each user's transmitted stream. Two common norm-based orderings are adopted, i.e., the streams are decoded in an ascending or a descending order. After V-BLAST, the relay retransmits the decoded information to the destination via a point-to-point FSO link in $m$ consecutive timeslots. Analytical expressions for the end-to-end outage probability and average symbol error probability of each user are derived, while closed-form asymptotic expressions are also presented. Capitalizing on the derived results, some engineering insights are manifested, such as the coding and diversity gain of each user, the impact of the pointing error displacement on the FSO link and the V-BLAST ordering effectiveness at the relay.
\end{abstract}

Index Terms-Decode-and-forward (DF), dual-hop communication, mixed RF/FSO systems, ordered successive interference cancellation (SIC), V-BLAST reception.

\section{INTRODUCTION}

D UAL-hop relaying has been widely adopted in the context of cooperative wireless communication systems, since the introduced spatial diversity can effectively extend the coverage area and mitigate the potentially severe channel fading [1]. One of the most popular relayed transmission strategies is established by employing the regenerative or the so-called decode-and-forward (DF) protocol. In this case, the overall end-to-end performance depends intimately on the decoding strategy and the appropriate signal processing at the relay [2]. An even more challenging scenario emerges in multiuser environments (i.e., in the presence of multiple source nodes). Such configurations, although beneficial in terms of

Manuscript received September 30, 2013; revised January 8, 2014. The editor coordinating the review of this paper and approving it for publication was M. Uysal.

Part of this paper has been accepted for publication at the IEEE International Conference on Communications (ICC), Sydney, Australia, June 2014.

N. I. Miridakis is with the Department of Informatics, University of Piraeus, 185 34, Piraeus, Greece, and with the Department of Computer Engineering, Technological Education Institute of Piraeus, 122 44, Aegaleo, Greece (e-mail: nikozm@unipi.gr).

M. Matthaiou is with the School of Electronics, Electrical Engineering and Computer Science, Queen's University Belfast, Belfast, BT3 9DT, U.K., and with the Department of Signals and Systems, Chalmers University of Technology, 412 96, Gothenburg, Sweden (e-mail: m.matthaiou@qub.ac.uk).

G. K. Karagiannidis is with the Department of Electrical and Computer Engineering, Aristotle University of Thessaloniki, 541 24, Thessaloniki, Greece, and with the Department of Electrical and Computer Engineering, Khalifa University, PO Box 127788, Abu Dhabi, UAE (e-mail: geokarag@ieee.org).

Digital Object Identifier 10.1109/TCOMM.2014.022314.130762 accessibility and coverage improvement, may introduce interuser interference, which degrades the overall performance unless properly compensated for [3]-[5].

In most relaying systems, radio frequency (RF) links are commonly used to support the entire end-to-end communication. Nevertheless, the scarcity of licensed spectrum resources in conjunction with the growing demands for highspeed communications, and the requirement for an increased accessibility from multiple users, represent some of the most crucial limitations of traditional RF/RF dual-hop communications. To this end, free-space optical (FSO) communications have attracted considerable research interest, due to their desirable features. More specifically, offering the potential of broadband communication capacity, since they operate at unlicensed optical wavelengths, FSO links represent a costeffective alternative and/or a complement to RF counterparts. Moreover, features such as flexibility, rapid deployment time, high security and robustness to RF interference have rendered FSO systems appealing for disaster recovery and military applications [6], [7]. We recall that both theoretical [8]-[11] and empirical research efforts [12]-[14] have manifested some of these useful aspects of the FSO systems.

Capitalizing on the aforementioned observations, the concept of mixed RF/FSO dual-hop relaying was recently introduced in the literature. First in [15] and then in [16], an analytical investigation of such topologies was conducted, when RF and FSO links are used for the first hop (source-torelay) and the second hop (relay-to-destination), respectively. It should be clarified that mixed RF/FSO systems refer to the case when RF transmission is used at one hop and FSO transmission at the other (i.e., different paths). On the other hand, hybrid RF/FSO systems describe the potential of parallel RF and FSO transmissions for the same path, (e.g., see [17][19]). However, [15] and [16] are limited to amplify-andforward (AF) relayed transmission, and, most importantly, assumed single-user communication scenarios. To the best of our knowledge, a corresponding performance analysis of DF relaying for the practical multiuser case, lacks from the open literature so far.

With this discussion in mind, the end-to-end performance of dual-hop DF relaying over mixed RF/FSO links in multiuser (multisource) environments is investigated. We consider single-antenna source nodes, a relay with multiple receive antennas and a single photo-aperture transmitter, and a destination node with a photo-detector. A direct application of the proposed configuration corresponds to the communication between a user and a base station (BS) for the source-to-relay RF link, and then from the BS to the backbone network (i.e., the last mile connection) for the relay-to-destination FSO link.

To mitigate interference caused by parallel multistream RF 
transmissions, the effective V-BLAST technique is utilized at the DF relay. Due to the complementary benefits of DF relaying and V-BLAST reception in terms of spatial diversity gain, dual-hop system configurations deploying V-BLAST relays have been extensively studied in the literature so far (e.g., see [20]-[25] and references therein). The V-BLAST technique consists of zero forcing (ZF) detection, followed by successive interference cancellation (SIC) [26]. It should be noted that in the conventional V-BLAST, symbol ordering is implemented exhaustively, prior to each SIC stage, to enhance the reception performance. However, such a sorting process induces high computational burden, which grows exponentially with the number of symbols to be detected [27]. Since the implementation cost of relays should be maintained as low as possible, we hereafter adopt a suboptimal, yet effective symbol ordering, which is established prior to only the primary SIC stage. After successive decoding, the relay forwards the multiple streams to the destination through a point-to-point FSO link in a number of consecutive timeslots.

The motivations behind such a system configuration are now outlined: (a) the concept of high-speed FSO links represents a fundamental motivation of the current work, since several RF streams can be multiplexed into a single FSO link [15]; (b) the aggregation of a maximum number of RF messages into a single FSO link can reach the maximum transmission capacity, thus it is a faster option as compared with the traditional RF/RF communication [16]; (c) interstream interference avoidance at the second hop relies on the point-to-point transmission over the relay-to-destination link, which, above all, represents a cost-efficient solution [16]; (d) since multiple streams are multiplexed into a single FSO link at the second hop, they can be efficiently transmitted in a number of consecutive timeslots, proportional to the number of streams. On the other hand, simultaneous RF transmissions at the first hop are established to reduce the end-to-end communication delay. Note that inter-stream interference can be effectively mitigated at the relay by adopting V-BLAST detection/decoding.

The contributions of this work can be summarized as follows:

- The scenario of the ordered ZF-SIC is studied by considering two popular norm-based orderings, namely, when symbols are sorted in a descending or an ascending order prior to the decoding process. Novel closed-form expressions for the probability density function (PDF) and the cumulative distribution function (CDF) of the respective SIC stages are derived. These expressions are performance metrics of the instantaneous signal-to-noise ratio (SNR) of the source-to-relay link for each user, when the RF signals undergo independent and identically distributed (i.i.d.) Rayleigh fading.

- New analytical expressions for the end-to-end outage probability and average symbol error probability (ASEP) of each user are derived. These analytical formulations are general, since they correspond to both V-BLAST orderings and account for pointing error impairments, atmospheric turbulence, path loss and the presence of white Gaussian noise in the FSO link.

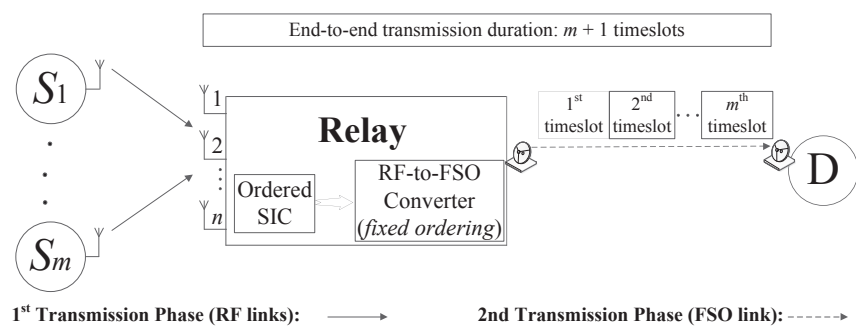

Fig. 1. The proposed system model with $\boldsymbol{S}_{i}(1 \leq i \leq m)$ and $\mathbf{D}$ denoting the $i$ th source node and the destination node, respectively.

- In the high SNR regime, simplified closed-form end-toend expressions for the outage probability and ASEP are given. Some useful insights, such as the diversity and the coding gain of each user are also obtained.

- From an engineering perspective, the impact of point-topoint displacements in the FSO link and the V-BLAST ordering effectiveness at the relay are discussed, over different system configuration scenarios and/or various channel fading conditions.

The rest of this paper is organized as follows: In Section II, the system model is presented in detail. The most important statistical properties of the source-to-relay links are derived in Section III. The analytical performance evaluation of the considered configuration is presented in Section IV and a corresponding asymptotic analysis is provided in Section V. Numerical results are presented in Section VI, while Section VII concludes the paper.

Notation: Vectors and matrices are represented by lowercase bold typeface and uppercase bold typeface letters, respectively. Also, $x_{i j}$, or $(\mathbf{X})_{i j}$, denote the element in the $i$ th row and $j$ th column of $\mathbf{X}$, while $x_{i}$ is the $i$ th vector coefficient of $\mathbf{x}$. The superscript $(.)^{H}$ denotes Hermitian transposition, $\|$. is the Euclidean vector norm, $\mathbb{E}[$.$] stands for the expectation$ operator, $\stackrel{\mathrm{d}}{=}$ represents equality in probability distributions and $o($.) stands for the Landau symbol (i.e., $f(x)=o(g(x))$, when $f(x) / g(x) \rightarrow 0$ as $x \rightarrow \infty)$.

\section{SYSTEM MODEL}

Consider a dual-hop communication system with $m$ singleantenna source nodes, a relay station equipped with $n \geq m$ antennas used for reception and a photo-aperture used for transmission, and a destination node with a photo-detector (c.f. Fig. 1). The source nodes transmit simultaneously to the destination via the intermediate relay, while the direct source-to-destination links are assumed to be absent due to strong propagation attenuation. The relay performs ordered SIC (i.e., V-BLAST), under the DF protocol, in $m$ consecutive stages. In principle, V-BLAST enables spatial multiplexing transmission, i.e., it can distinguish the received streams from different users and/or antennas with the aid of spatial structures (individual spatial signatures) of the signals to be detected [28]. Afterwards, the successively (in $m$ stages) decoded information is retransmitted to the destination in $m$ consecutive timeslots (corresponding to the $m$ source nodes) via a point-to-point FSO link. One of the many potential applications of this topology, is when considering a building 
floor within a femtocell of a heterogeneous network (e.g, an LTE-A setup), where users want to access the FSO backbone via an intermediate relay, placed at one of the cornens of that floor.

The end-to-end communication is established in two transmission phases; one for the source-to-relay link(s) and one for the relay-to-destination link. The former phase occurs via $m$ RF chains, while a point-to-point FSO link is used for the latter phase. For the second hop, a quasi-static channel is assumed, i.e., the channel fading remains unchanged over the duration of $m$ timeslots (e.g., the duration of the second transmission phase), while it may change afterwards.

Assuming perfect channel state information (CSI) at the relay, the end-to-end SNR for DF dual-hop systems reads as [29]

$$
\gamma_{e 2 e}^{(i)} \triangleq \min \left\{\gamma_{1}^{(i)}, \gamma_{2}\right\}
$$

where $\gamma_{e 2 e}^{(i)}, \gamma_{1}^{(i)}$ and $\gamma_{2}$ represent the instantaneous end-to-end SNR of the $i$ th source node $(1 \leq i \leq m)$, the SNR between the $i$ th source node and the relay, and the SNR between the relay and the destination (identical in all timeslots due to the quasi-static channel assumption), respectively.

The RF transmission in the first phase can be considered as a distributed multiple input-multiple output (MIMO) communication system with $m$ transmit and $n \geq m$ receive antennas. Then, the standard input-output relation of the received signal can be represented as

$$
\mathbf{r}=\mathbf{G s}+\mathbf{w},
$$

where $\mathbf{r} \in \mathbb{C}^{n \times 1}, \mathbf{s} \in \mathbb{C}^{m \times 1}$ and $\mathbf{w} \in \mathbb{C}^{n \times 1}$ denote the received, the transmit and the circularly symmetric Gaussian noise signal vectors, respectively. Moreover, G $\in \mathbb{C}^{n \times m}$ corresponds to the channel fading matrix, including i.i.d. complex Gaussian coefficients with zero mean and unit variance (i.e., a Rayleigh flat fading scenario). The i.i.d. statistical assumption can be ensured by considering almost equallyspaced source nodes with respect to the relay station or a preestablished perfect power control scheme. Such a requirement preserves identical signal propagation effects (e.g., an equal input average SNR for every transmission), which, in turn, facilitates the analytical tractability of our study. Note that the assumption of i.i.d. fading is detrimental for the subsequent performance analysis. Interestingly, the presented results can serve as upper bounds for the more general case of correlated Rayleigh fading.

\section{A. V-BLAST decoding at the relay}

The classical V-BLAST is performed in three main steps, namely, the (optional) symbol ordering that aims to enhance the overall reception performance, the interference nulling via ZF from the yet-to-be detected symbols, and the interference cancellation from the already detected symbols. These steps are performed in a number of consecutive stages, until all given symbols are successfully decoded. In the optimal VBLAST, the symbol ordering is performed at every SIC stage, thereby demanding an exhaustive search among $m$ ! possible ordering combinations [30]. In the rest of the paper, and similar to [31], we turn our focus on the suboptimal, yet more efficient scheme (in terms of computational complexity), in which the appropriate ordering is implemented only at the primary SIC stage. Hence, there are only $m(m+1) / 2-1$ possible orderings required, which represent a remarkable computational gain over the clear-optimal ordering, especially for high $m$ values, e.g., when $m \geq 4$. Moreover, it has been shown that there is no performance degradation between the suboptimal and optimal ordering with respect to diversity gain, but only a slight penalty on the coding gain [32]. To this end, we elaborate on two suboptimal ordering approaches, which are based on the channel gains and are established prior to the initial first SIC stage. Typically, the ordering procedure is equivalent to mapping a channel matrix into a corresponding permutation matrix. Thus, we define the permuted (based on the appropriate ordering) matrix $\mathbf{H} \triangleq \mathbf{G P}$, where $\mathbf{P} \in \mathbb{R}^{m \times m}$ is the permutation matrix. ${ }^{1}$

The interference nulling can be efficiently implemented by applying the QR decomposition on a given channel matrix, by adopting either the Gram-Schmidt projections [33] or the Householder transformations [31]. It is noteworthy that QR decomposition is widely adopted in ZF equalizers, since it provides computational complexity savings [34]. In what follows, and without loss of generality, the Householder transformations are considered. The standard QR decomposition of the permuted channel matrix is developed by left multiplying H with $m$ Householder matrices successively.

At the receiver end (the relay), it holds that

$$
\tilde{\mathbf{r}}=\mathbf{R s}+\mathbf{Q}^{H} \mathbf{w}
$$

where $\tilde{\mathbf{r}}=\mathbf{Q}^{H} \mathbf{r}, \mathbf{Q}$ is a $n \times n$ unitary matrix (with its columns representing the orthonormal $\mathrm{ZF}$ nulling vectors) and $\mathbf{R}$ is an $n \times m$ upper triangular matrix. Assuming perfect CSI at the receiver and, hence, by neglecting the error propagation effect between the consecutive SIC stages, the instantaneous SNR per stream is given as [31], [35] $\gamma_{1}^{(i)}=r_{i i}^{2}$, where $r_{i j}$ stands for the $(i, j)$ th entry of $\mathbf{R}$.

\section{B. Some useful statistical formulae}

We now summarize some algebraic results, which will be useful for the following analysis.

Remark 1: Given a set of $m$ i.i.d. random variables (RVs) arranged in an ascending order, i.e., $x_{1}<x_{2}<\ldots<x_{m}$, then the corresponding PDF of $x_{i}$ is expressed as [36]

$$
f_{x_{i}}(x) \triangleq \frac{1}{B(i, m-i+1)} F_{x}(x)^{i-1}\left(1-F_{x}(x)\right)^{m-i} f_{x}(x) \text {, }
$$

where $f_{x_{i}}(),. F_{x}(),. f_{x}($.$) and B(.,$.$) represent the PDF of$ the $i$ th ordered RV, the CDF of the unordered RV, the PDF of the unordered RV and the Beta function [37, Eq. (8.384.1)], respectively. In the case when the RVs are arranged in a descending order, i.e., $x_{1}>x_{2}>\ldots>x_{m}$, the PDF of $x_{i}$ becomes [36]

$$
f_{x_{i}}(x) \triangleq \frac{1}{B(i, m-i+1)} F_{x}(x)^{m-i}\left(1-F_{x}(x)\right)^{i-1} f_{x}(x) \text {. }
$$

\footnotetext{
${ }^{1}$ In the case of fixed ordering (i.e., no ordering), $\mathbf{H}=\mathbf{G}$ and $\mathbf{P}=\mathbf{I}_{m}$, where $\mathbf{I}_{m}$ stands for the identity matrix of size $m$.
} 
Remark 2: Let $\mathbf{x}$ denote a complex-valued RV vector. Then, $\mathbf{x}$ is isotropically distributed if its distribution is invariant under rotations and reflections [38]. Particularly, $\mathbf{x}$ is isotropic if it holds that $\mathbf{x} \stackrel{\mathrm{d}}{=} \mathbf{Y x}$, for any unitary matrix $\mathbf{Y}$. Furthermore, the direction vector $\mathbf{v} \triangleq \mathbf{x} /\|\mathbf{x}\|$ and its Euclidean norm $\|\mathbf{x}\|$ are statistically independent [38, Theorem 1.5.5]. Thereby, $\mathbf{x}$ is still isotropically distributed regardless if its norm is under some conditional constraint [31].

Lemma 1: Under Rayleigh fading conditions, it holds that

$$
\left\|\mathbf{h}_{i}(i: n)\right\|^{2} \stackrel{\mathrm{d}}{=}\left\|\mathbf{r}_{i}(i: n)\right\|^{2},
$$

where $\mathbf{h}_{i}$ denotes the $i$ th column of $\mathbf{H}$. Note that $\left\|\mathbf{x}_{i}(i: n)\right\|$ consists of the last $n-i+1$ coefficients of a given $n$ dimensional $\mathbf{x}$ vector, while $\left\|\mathbf{x}_{i}\right\| \triangleq\left\|\mathbf{x}_{i}(1: n)\right\|$.

Proof: The proof is obtained by using Remark 2 in conjunction with [31, Lemma V.3].

Moreover, it is worth mentioning that $\left\|\mathbf{r}_{i}(i: n)\right\|^{2}=r_{i i}^{2}$ and, thus, $r_{i i}^{2} \stackrel{\mathrm{d}}{=}\left\|\mathbf{h}_{i}(i: n)\right\|^{2}$, due to the upper triangular structure of $\mathbf{R}$. In the following, and for notational simplicity, we introduce the auxiliary variables $r_{i i}^{2} \triangleq \mathcal{X}_{i}$ and $\left\|\mathbf{h}_{i}\right\|^{2} \triangleq \mathcal{Y}_{i}$.

Remark 3: The PDF of $\mathcal{X}_{i}$ conditioned on $\mathcal{Y}_{i}, 2 \leq i \leq m$, is expressed as [31, Eq. (47)]

$$
f_{\mathcal{X}_{i} \mid \mathcal{Y}_{i}}(x \mid y)=\frac{x^{n-i}(y-x)^{i-2}}{y^{n-1} B(n-i+1, i-1)}, \quad 0 \leq x \leq y .
$$

As previously mentioned, V-BLAST may enforce symbol ordering or not. Since the main rationale of this work is the analytical description of the ordered V-BLAST (or the ordered SIC), we, hereafter, focus our interest only to the ordered case.

Remark 4: Although $m$ ! different symbol (norm-based) orderings may occur, (6) holds for any ordering when $i \geq 2$, as stated in Remark 2. In the case of $\mathcal{X}_{1}$, we have that $\mathcal{X}_{1}=\left\|\mathbf{h}_{1}(1: n)\right\|^{2}=\mathcal{Y}_{1}$. Thus, the unconditional PDF of the last SIC stage (i.e., the first layer) is given as $f_{\mathcal{X}_{1}}(x)=f_{\mathcal{Y}_{1}}(x)$ and is directly affected by the precise ordering strategy.

The forward decoding is adopted into this work and, therefore, the first SIC stage corresponds to the last decoding layer of the processing matrix (from the left to the right). Following the same philosophy, the $i$ th decoding layer corresponds to the $(m-i+1)$ th SIC stage. Note that the terms decoding layer and SIC stage will be interchangeably used in the rest of this paper.

\section{Relay-to-Destination transmission: FSO link}

Consider a point-to-point FSO link with indirect modulation/direct detection (IM/DD), which is established for the relay-to-destination communication. The FSO signal is affected by pointing error impairments, path loss, atmospheric turbulence and white Gaussian noise. The corresponding SNR is assumed to experience Gamma-Gamma fading with a PDF given by [16], [39]

$$
f_{\gamma_{2}}(x)=\frac{\xi^{2}}{2 x \Gamma(\mathcal{A}) \Gamma(\mathcal{B})} G_{1,3}^{3,0}\left[\mathcal{A B} \sqrt{\frac{x}{\bar{\gamma}_{2}}} \mid \begin{array}{c}
\xi^{2}+1 \\
\xi^{2}, \mathcal{A}, \mathcal{B}
\end{array}\right],
$$

where $\xi$ is the ratio between the equivalent beam radius and the pointing error displacement standard deviation at the destination, $\mathcal{A}$ and $\mathcal{B}$ are the fading parameters related to the atmospheric turbulence conditions (with lower values indicating severe turbulence conditions [39, Eq. (3)]). Also, $\Gamma(\alpha) \triangleq \int_{0}^{\infty} t^{\alpha-1} \exp (-t) d t$ denotes the Gamma function [37, Eq. (8.310.1)], $\bar{\gamma}_{2} \triangleq \mathbb{E}[x] E_{s} / N_{0}$ is the average SNR of the relay-to-destination link and $E_{s}, N_{0}$ denote the energy per symbol and the single-sided power spectral density of the noise, respectively. Finally, $G[$.$] represents the Meijer's-$ $G$ function [37, Eq. (9.301)].

\section{Source-to-Relay Statistical Analysis}

In this section, some important statistical features (namely, the PDF and the CDF) of each SIC stage, which is performed at the relay, are analytically investigated. Two very popular norm ordering schemes are closely examined; the rate efficient approach [31] and the error efficient approach [40]. The former corresponds to an ascending symbol ordering (i.e., the weakest symbol is decoded first and the strongest symbol is decoded last), in order to improve the total spectral efficiency at the cost of error efficiency loss [31]. On the other hand, the latter stands for the classical V-BLAST ordering, firstly proposed by Foschini et al. in [40], where the symbols are decoded in a descending order of magnitude, i.e., the strongest symbol is decoded first while the weakest symbol is decoded last. Such an ordering has shown very good results in terms of error rates.

Assuming that the received signal undergoes i.i.d. Rayleigh fading, the unordered squared column norms follow a chisquared distribution with $2 n$ degrees of freedom. Moreover, the PDF of the $i$ th ordered squared column norm, denoted as $f_{\mathcal{Y}_{i}}(x)$, can be derived by substituting the standard chisquared PDF/CDF expressions into (3) or (4), depending on which ordering approach is followed.

\section{A. The rate efficient ordering}

Theorem 1: The PDF of the SNR at the $i$ th decoding layer, when $2 \leq i \leq m$, is expressed as

$$
\begin{aligned}
& f_{\mathcal{X}_{i}}(x)=\Xi(n, m, i) \\
& \times \sum_{r=0}^{i+\phi-j-2} \frac{(i+\phi-j-2) ! x^{r+n+j-i} \exp \left(-\frac{\Delta_{2}}{\bar{\gamma}_{1}} x\right)}{r !\left(\frac{\Delta_{2}}{\bar{\gamma}_{1}}\right)^{i+\phi-j-r-1}},
\end{aligned}
$$

where

$$
\begin{aligned}
& \Xi(n, m, i) \triangleq \\
& \sum_{j=0}^{i-2} \sum_{l=0}^{\Delta_{1}} \sum_{p_{1}=0}^{\Delta_{3}} \sum_{p_{2}=0}^{p_{1}} \ldots \sum_{p_{n-1}=0}^{p_{n-2}} \prod_{t=1}^{n-1}\left[\frac{1}{\left(p_{t-1}-p_{t}\right) !(t !)^{p_{t}-p_{t+1}}}\right] \\
& \times \frac{(-1)^{j+l}\left(\Delta_{3}\right) !\left(\begin{array}{c}
i-2 \\
j
\end{array}\right)\left(\begin{array}{c}
\Delta_{1} \\
l
\end{array}\right)}{p_{n-1} ! \bar{\gamma}_{1}^{n+\phi} B(n-i+1, i-1) B(m-i+1, i)(n-1) !},
\end{aligned}
$$

while $\bar{\gamma}_{1} \triangleq \mathbb{E}[x] E_{s} / N_{0}$ is the average SNR of the source-torelay link. Also, $\Delta_{1} \triangleq m-i, \Delta_{2} \triangleq i+l, \Delta_{3} \triangleq \Delta_{2}-1$, $p_{0}=\Delta_{3}, p_{n}=0$ and $\phi \triangleq \sum_{q=1}^{n-1} p_{q}$.

Proof: The proof is given in Appendix A. 
Corollary 1: The CDF of the SNR at the $i$ th decoding layer, when $2 \leq i \leq m$, is expressed as

$$
\begin{aligned}
F_{\mathcal{X}_{i}}(x) & =\Xi(n, m, i) \sum_{r=0}^{i+\phi-j-2} \frac{(i+\phi-j-2) !}{r !\left(\frac{\Delta_{2}}{\bar{\gamma}_{1}}\right)^{n+\phi}} \\
& \times \gamma\left(r+n+j-i+1, \frac{\Delta_{2}}{\bar{\gamma}_{1}} x\right) \\
& =\Xi(n, m, i) \sum_{r=0}^{i+\phi-j-2} \frac{(i+\phi-j-2) !(r+n+j-i) !}{r !\left(\frac{\Delta_{2}}{\bar{\gamma}_{1}}\right)^{n+\phi}} \\
& \times\left[1-\exp \left(-\frac{\Delta_{2}}{\bar{\gamma}_{1}} x\right)^{r+n+j-i} \sum_{s=0}^{r} \frac{x^{s}}{s !\left(\frac{\Delta_{2}}{\bar{\gamma}_{1}}\right)^{-s}}\right],
\end{aligned}
$$

where $\gamma(\alpha, x) \triangleq \int_{0}^{x} t^{\alpha-1} \exp (-t) d t$ denotes the lower incomplete Gamma function [37, Eq. (8.350.1)].

Theorem 2: The PDF of the SNR at the 1st decoding layer is expressed as

$$
f_{\mathcal{X}_{1}}(x)=\Psi(n, m) x^{n+\phi-1} \exp \left(-\frac{(j+1)}{\bar{\gamma}_{1}} x\right),
$$

where

$$
\begin{aligned}
& \Psi(n, m) \triangleq \sum_{j=0}^{m-1} \sum_{p_{1}=0}^{j} \sum_{p_{2}=0}^{p_{1}} \cdots \sum_{p_{n-1}=0}^{p_{n-2}} \\
& \times \prod_{t=1}^{n-1}\left[\frac{1}{\left(p_{t-1}-p_{t}\right) !(t !)^{p_{t}-p_{t+1}}}\right] \frac{\left(\begin{array}{c}
m-1 \\
j
\end{array}\right)(-1)^{j}(j) ! m}{p_{n-1} ! \bar{\gamma}_{1}^{n+\phi}(n-1) !}
\end{aligned}
$$

while in this case it holds that $p_{0}=j, p_{n}=0$ and $\phi \triangleq$ $\sum_{q=1}^{n-1} p_{q}$.

Proof: The proof is relegated in Appendix B.

Corollary 2: The CDF of the SNR at the 1st decoding layer is expressed as

$$
\begin{aligned}
F_{\mathcal{X}_{1}}(x) & =\Psi(n, m) \frac{\gamma\left(n+\phi, \frac{(j+1)}{\bar{\gamma}_{1}} x\right)}{\left(\frac{j+1}{\bar{\gamma}_{1}}\right)^{n+\phi}} \\
& =\Psi(n, m) \frac{(n+\phi-1) !}{\left(\frac{j+1}{\bar{\gamma}_{1}}\right)^{n+\phi}} \\
& \times\left(1-\exp \left(-\frac{(j+1)}{\bar{\gamma}_{1}} x\right) \sum_{s=0}^{n+\phi-1} \frac{x^{s}}{s !\left(\frac{j+1}{\bar{\gamma}_{1}}\right)^{-s}}\right) .
\end{aligned}
$$

\section{B. The error efficient ordering}

As previously mentioned, the error efficient approach represents the classical V-BLAST ordering.

Corollary 3: The PDF and the CDF of the SNR at the $i$ th decoding layer, when $2 \leq i \leq m$, can be directly obtained from (8) and (10), respectively, by applying the following substitutions

$$
\begin{aligned}
& \Delta_{1} \triangleq i-1, \\
& \Delta_{2} \triangleq m+l-i+1 .
\end{aligned}
$$

Moreover, the PDF and CDF of the SNR at the 1st decoding layer are obtained from (11) and (13), respectively, by setting $j=m-1$ and $(-1)^{j}=1$.

Proof: The proof is provided in Appendix C.

\section{End-TO-End System PERformancE}

Some of the most important metrics in digital communications are analytically studied and evaluated into this section, namely the outage probability and ASEP. Both the rate efficient and the error efficient ordering approaches are considered.

\section{A. Outage Probability}

The outage probability is defined as the probability that the SNR falls below a certain threshold value, $\gamma_{\text {th }}$, such that

$$
P_{\text {out }}^{(i)}\left(\gamma_{\text {th }}\right)=\operatorname{Pr}\left\{\gamma_{e 2 e}^{(i)} \leq \gamma_{\text {th }}\right\}, \quad 1 \leq i \leq m
$$

1) The rate efficient approach:

Theorem 3: The outage probability of $\gamma_{e 2 e}$ is expressed as

$$
\begin{aligned}
& P_{\text {out }}^{(i)}\left(\gamma_{\mathrm{th}}\right)=1-\Xi(n, m, i) \sum_{r=0}^{i+\phi-j-2} \sum_{k=0}^{r+n+j-i} \\
& \times \frac{(i+\phi-j-2) !(r+n+j-i) ! \xi^{2} x^{k} \exp \left(-\frac{\Delta_{2}}{\bar{\gamma}_{1}} x\right)}{r ! k ! \Gamma(\mathcal{A}) \Gamma(\mathcal{B})\left(\frac{\Delta_{2}}{\bar{\gamma}_{1}}\right)^{\phi+n-k}} \\
& \times G_{2,4}^{4,0}\left[\frac{\mathcal{A} \mathcal{B} \sqrt{x}}{\sqrt{\bar{\gamma}_{2}}} \mid \begin{array}{c}
\xi^{2}+1,1 \\
0, \xi^{2}, \mathcal{A}, \mathcal{B}
\end{array}\right], \quad 2 \leq i \leq m
\end{aligned}
$$

and

$$
\begin{aligned}
& P_{\text {out }}^{(1)}\left(\gamma_{\mathrm{th}}\right)=1-\Psi(n, m) \sum_{k=0}^{n+\phi-1} \frac{(n+\phi-1) ! \xi^{2}}{k ! \Gamma(\mathcal{A}) \Gamma(\mathcal{B})\left(\frac{j+1}{\bar{\gamma}_{1}}\right)^{n+\phi-k}} \\
& \times x^{k} \exp \left(-\frac{j+1}{\bar{\gamma}_{1}} x\right) G_{2,4}^{4,0}\left[\frac{\mathcal{A B} \sqrt{x}}{\sqrt{\bar{\gamma}_{2}}} \mid \begin{array}{c}
\xi^{2}+1,1 \\
0, \xi^{2}, \mathcal{A}, \mathcal{B}
\end{array}\right] . \quad(17)
\end{aligned}
$$

Proof: The proof is given in Appendix D.

Corollary 4: For the (ideal) non-pointing error case, the outage probability of $\gamma_{e 2 e}$ is given by

$$
\begin{aligned}
& P_{\text {out }}^{(i)}\left(\gamma_{\mathrm{th}}\right)=1-\Xi(n, m, i) \sum_{r=0}^{i+\phi-j-2} \sum_{k=0}^{r+n+j-i} \\
& \times \frac{(i+\phi-j-2) !(r+n+j-i) ! x^{k} \exp \left(-\frac{\Delta_{2}}{\bar{\gamma}_{1}} x\right)}{r ! k ! \Gamma(\mathcal{A}) \Gamma(\mathcal{B})\left(\frac{\Delta_{2}}{\bar{\gamma}_{1}}\right)^{\phi+n-k}} \\
& \times G_{1,3}^{3,0}\left[\frac{\mathcal{A} \mathcal{B} \sqrt{x}}{\sqrt{\bar{\gamma}_{2}}} \mid \begin{array}{c}
0, \mathcal{A}, \mathcal{B}
\end{array}\right], \quad 2 \leq i \leq m,
\end{aligned}
$$

and

$$
\begin{aligned}
& P_{\text {out }}^{(1)}\left(\gamma_{\text {th }}\right)=1-\Psi(n, m) \sum_{k=0}^{n+\phi-1} \frac{(n+\phi-1) !}{k ! \Gamma(\mathcal{A}) \Gamma(\mathcal{B})\left(\frac{j+1}{\bar{\gamma}_{1}}\right)^{n+\phi-k}} \\
& \times x^{k} \exp \left(-\frac{j+1}{\bar{\gamma}_{1}} x\right) G_{1,3}^{3,0}\left[\frac{\mathcal{A} \mathcal{B} \sqrt{x}}{\sqrt{\bar{\gamma}_{2}}} \mid \begin{array}{c}
1 \\
0, \mathcal{A}, \mathcal{B}
\end{array}\right] .
\end{aligned}
$$

Proof: Taking $\xi \rightarrow \infty$, we have that $\xi^{2}+1 \approx \xi^{2}$. Thus, applying [37, Eq. (9.31.1)] into (16) and (17) yields (18) and (19), respectively. 
2) The error efficient approach: It is trivial to show that quite similar expressions hold also for the error efficient ordering approach, according to the modifications indicated in Corollary 3. The corresponding results are omitted though, for the sake of brevity.

\section{B. ASEP}

The end-to-end ASEP of the $i$ th link, denoted as $\overline{P s}_{i}$, can be directly evaluated, according to [42], [43]

$$
\overline{P s}_{i}=\frac{\alpha \sqrt{\beta}}{2 \sqrt{\pi}} \int_{0}^{\infty} \frac{\exp (-\beta x)}{\sqrt{x}} F_{\gamma_{e 2 e}}^{(i)}(x) d x
$$

where $\alpha$ and $\beta$ are specific constants that define the modulation type, e.g., $(\alpha, \beta)=(1,1)$ for BPSK constellation alphabets.

\section{1) The rate efficient approach:}

Theorem 4: The end-to-end ASEP of the $i$ th link, when $2 \leq$ $i \leq m$, is expressed as

$$
\begin{aligned}
& \overline{P s}_{i}=\frac{\alpha}{2}\left\{1-\Xi(n, m, i) \sum_{r=0}^{i+\phi-j-2} \sum_{k=0}^{r+n+j-i}\right. \\
& \times \frac{(i+\phi-j-2) !(r+n+j-i) ! \xi^{2} \sqrt{\beta} 2^{\mathcal{A}+\mathcal{B}-3}}{r ! k ! \Gamma(\mathcal{A}) \Gamma(\mathcal{B})\left(\frac{\Delta_{2}}{\bar{\gamma}_{1}}\right)^{\phi+n-k} \pi^{3 / 2}\left(\frac{\Delta_{2}}{\bar{\gamma}_{1}}+\beta\right)^{k+1 / 2}} \\
& \left.\times G_{3,6}^{6,1}\left[\frac{(\mathcal{A B})^{2}}{16 \bar{\gamma}_{2}\left(\frac{\Delta_{2}}{\bar{\gamma}_{1}}+\beta\right)} \mid \frac{\xi^{2}}{2}, 0, \frac{\mathcal{A}}{2}, \frac{\mathcal{A}+1}{2}, \frac{\mathcal{B}}{2}, \frac{\mathcal{B}+1}{2}\right]\right\},
\end{aligned}
$$

and the corresponding ASEP, when $i=1$, is equal to

$$
\begin{aligned}
& \overline{P s}_{1}=\frac{\alpha}{2}\left\{1-\Psi(n, m) \sum_{k=0}^{n+\phi-1}\right. \\
& \times \frac{(n+\phi-1) ! \xi^{2} \sqrt{\beta} 2^{\mathcal{A}+\mathcal{B}-3}}{k ! \Gamma(\mathcal{A}) \Gamma(\mathcal{B})\left(\frac{j+1}{\bar{\gamma}_{1}}\right)^{\phi+n-k} \pi^{3 / 2}\left(\frac{j+1}{\bar{\gamma}_{1}}+\beta\right)^{k+1 / 2}} \\
& \left.\times G_{3,6}^{6,1}\left[\frac{(\mathcal{A B})^{2}}{16 \bar{\gamma}_{2}\left(\frac{j+1}{\bar{\gamma}_{1}}+\beta\right)} \mid \begin{array}{c}
\frac{1}{2}-k, \frac{\xi^{2}}{2}+1,1 \\
\frac{\xi^{2}}{2}, 0, \frac{\mathcal{A}}{2}, \frac{\mathcal{A}+1}{2}, \frac{\mathcal{B}}{2}, \frac{\mathcal{B}+1}{2}
\end{array}\right]\right\} .
\end{aligned}
$$

Proof: Plugging (16) or (17) into (20), thereafter utilizing [44, Eq. (2.24.1.1)] and after performing some straightforward manipulations, gives (21) or (22), respectively.

Corollary 5: In the case of non-pointing errors, the end-to-end
ASEP of the $i$ th link, when $2 \leq i \leq m$, is expressed as

$$
\begin{aligned}
& \overline{P s}_{i}=\frac{\alpha}{2}\left\{1-\Xi(n, m, i) \sum_{r=0}^{i+\phi-j-2} \sum_{k=0}^{r+n+j-i}\right. \\
& \times \frac{(i+\phi-j-2) !(r+n+j-i) ! \sqrt{\beta} 2^{\mathcal{A}+\mathcal{B}-3}}{r ! k ! \Gamma(\mathcal{A}) \Gamma(\mathcal{B})\left(\frac{\Delta_{2}}{\bar{\gamma}_{1}}\right)^{\phi+n-k} \pi^{3 / 2}\left(\frac{\Delta_{2}}{\bar{\gamma}_{1}}+\beta\right)^{k+1 / 2}} \\
& \times G_{2,5}^{5,1}\left[\frac{(\mathcal{A B})^{2}}{16 \bar{\gamma}_{2}\left(\frac{\Delta_{2}}{\bar{\gamma}_{1}}+\beta\right)} \mid 0, \frac{\mathcal{A}}{2}, \frac{\left.\left.\frac{\mathcal{A}+1}{2}, \frac{\mathcal{B}}{2}, \frac{\mathcal{B}+1}{2}\right]\right\},}{}\right.
\end{aligned}
$$

and the corresponding ASEP, when $i=1$, becomes

$$
\begin{aligned}
& \overline{P s}_{1}=\frac{\alpha}{2}\left\{1-\Psi(n, m) \sum_{k=0}^{n+\phi-1}\right. \\
& \times \frac{(n+\phi-1) ! \sqrt{\beta} 2^{\mathcal{A}+\mathcal{B}-3}}{k ! \Gamma(\mathcal{A}) \Gamma(\mathcal{B})\left(\frac{j+1}{\bar{\gamma}_{1}}\right)^{\phi+n-k} \pi^{3 / 2}\left(\frac{j+1}{\bar{\gamma}_{1}}+\beta\right)^{k+1 / 2}} \\
& \left.\times G_{2,5}^{5,1}\left[\frac{(\mathcal{A B})^{2}}{16 \bar{\gamma}_{2}\left(\frac{j+1}{\bar{\gamma}_{1}}+\beta\right)} \mid 0, \frac{\mathcal{A}}{2}, \frac{\frac{1}{2}-k+1}{2}, \frac{\mathcal{B}}{2}, \frac{\mathcal{B}+1}{2}\right]\right\} .
\end{aligned}
$$

2) The error efficient approach: In this case, ASEP can be directly obtained from (21) and (22), by following the substitutions indicated in Corollary 3.

Notably, both the end-to-end outage probability and ASEP are obtained via finite sums of Meijer's- $G$ functions, which represent standard built-in functions in most popular mathematical software packages. Hence, such expressions can be very rapidly and efficiently computed. Furthermore, these expressions can be simplified to finite sum series of the more familiar generalized hypergeometric functions [37, Eq. (9.14.1)], according to [37, Eq. (9.303)].

As an illustrative example, consider the most simple scenario, where only two source nodes are used and the relay is equipped with two antennas, i.e., when $m=n=2$. Based on (16), it is not difficult to show that the end-to-end outage probability at the second decoding layer (or the first SIC stage), in the case of the rate efficient ordering, becomes

$$
\begin{aligned}
& P_{\text {out }}^{(2)}\left(\gamma_{\text {th }}\right)=1-\exp \left(-\frac{2 \gamma_{\text {th }}}{\bar{\gamma}_{1}}\right) \frac{\left(2 \bar{\gamma}_{1}+\gamma_{\text {th }}\right) \xi^{2}}{2 \bar{\gamma}_{1} \Gamma(\mathcal{A}) \Gamma(\mathcal{B})}
\end{aligned}
$$

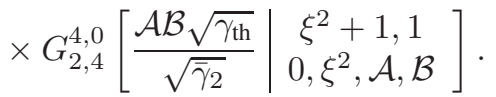

Correspondingly, based on (17), the end-to-end outage probability at the first decoding layer (or the second SIC stage) simplifies to

$$
\begin{aligned}
& P_{\text {out }}^{(1)}\left(\gamma_{\text {th }}\right)= \\
& 1-\exp \left(-\frac{2 \gamma_{\text {th }}}{\bar{\gamma}_{1}}\right) G_{2,4}^{4,0}\left[\frac{\mathcal{A} \mathcal{B} \sqrt{\gamma_{\text {th }}}}{\sqrt{\bar{\gamma}_{2}}} \mid \begin{array}{c}
\xi^{2}+1,1 \\
0, \xi^{2}, \mathcal{A}, \mathcal{B}
\end{array}\right] \\
& \times \frac{\left(\exp \left(\frac{\gamma_{\text {th }}}{\bar{\gamma}_{1}}\right) 2 \bar{\gamma}_{1}\left(\bar{\gamma}_{1}+\gamma_{\text {th }}\right)-\gamma_{\text {th }}^{2}-2 \gamma_{\text {th }} \bar{\gamma}_{1}-\bar{\gamma}_{1}^{2}\right) \xi^{2}}{\bar{\gamma}_{1}^{2} \Gamma(\mathcal{A}) \Gamma(\mathcal{B})} .
\end{aligned}
$$


Following the same methodology and invoking Theorems 3 and 4, similar expressions can be obtained for the error efficient ordering approach and/or the non-pointing error case.

\section{ASYMPTOTIC ANALYSIS}

In the high SNR regime, the previously described analytical expressions admit a more amenable formulation. Therefore, some useful insights can be obtained regarding the performance of each end-to-end link, such as the coding and the diversity gain. We define $\mathcal{P}_{c_{i}}, \mathcal{P}_{d_{i}}, \mathcal{S}_{c_{i}}$ and $\mathcal{S}_{d_{i}}$ the outage coding gain, the outage diversity gain, the ASEP coding gain and the ASEP diversity gain of the $i$ th end-to-end link, respectively [45]. First, let

$$
\lim _{\bar{\gamma}_{1}, \bar{\gamma}_{2} \rightarrow \infty} F_{\gamma_{e 2 e}}^{(i)}(x)=1-\lim _{\bar{\gamma}_{1} \rightarrow \infty} \mathcal{I}_{1} \lim _{\bar{\gamma}_{2} \rightarrow \infty} \mathcal{I}_{2},
$$

denote the CDF of the $i$ th end-to-end link in the asymptotically high SNR regime, where $\mathcal{I}_{1}$ and $\mathcal{I}_{2}$ are defined in Appendix D.1. Then, it is not difficult to show that $\lim _{\bar{\gamma}_{2} \rightarrow \infty} \mathcal{I}_{2}=1$, since it holds from (D.4) that [37, Eq. (9.303)]

$$
\lim _{\bar{\gamma}_{2} \rightarrow \infty} G_{2,4}^{4,0}\left[\frac{\mathcal{A B} \sqrt{x}}{\sqrt{\bar{\gamma}_{2}}} \mid \begin{array}{c}
\xi^{2}+1,1 \\
0, \xi^{2}, \mathcal{A}, \mathcal{B}
\end{array}\right]=\frac{\Gamma(\mathcal{A}) \Gamma(\mathcal{B})}{\xi^{2}} .
$$

The above expression stems from expanding the Meijer's- $G$ function in a finite sum series representation according to [37, Eq. (9.303)], and thereafter keeping only the most dominant sum term. Hence, we have that

$$
\lim _{\bar{\gamma}_{1}, \bar{\gamma}_{2} \rightarrow \infty} F_{\gamma_{e 2 e}}^{(i)}(x)=1-\lim _{\bar{\gamma}_{1} \rightarrow \infty} \mathcal{I}_{1}=\lim _{\bar{\gamma}_{1} \rightarrow \infty} F_{\gamma_{1}}^{(i)}(x) .
$$

Remark 5: It is obvious from (28), and it will be verified in the following, that in the asymptotically high average SNR regime, the asymptotic gains of $\gamma_{e 2 e}$ are only affected by the decoding strategy that is implemented at the relay.

Such gains are explicitly given subsequently, when both the rate efficient and the error efficient ordering approaches are implemented in the V-BLAST decoding at the relay.

\section{A. The rate efficient approach}

Proposition 1: The asymptotic CDF (i.e., the asymptotic outage probability) at the $i$ th decoding layer (or the $i$ th endto-end link), when $2 \leq i \leq m$, is derived as

$$
\begin{aligned}
& F_{\gamma_{e 2 e}}^{(i)}\left(\gamma_{\mathrm{th}}\right)= \\
& \frac{m !\left(\frac{\gamma_{\mathrm{th}}}{\bar{\gamma}_{1}}\right)^{n-i+1}}{(i-1) !(m-i) !(n-i+1) ! i^{i-1}}+o\left(\bar{\gamma}_{1}^{-(n-i+1)}\right) .
\end{aligned}
$$

The corresponding asymptotic $\mathrm{CDF}$ at the 1 st decoding layer is given by

$$
F_{\gamma_{e 2 e}}^{(1)}\left(\gamma_{\mathrm{th}}\right)=\frac{1}{(n !)^{m}}\left(\frac{\gamma_{\mathrm{th}}}{\bar{\gamma}_{1}}\right)^{m n}+o\left(\bar{\gamma}_{1}^{-m n}\right) .
$$

Proof: The proof is relegated in Appendix E.

Proposition 2: The asymptotic ASEP at the $i$ th decoding layer, when $2 \leq i \leq m$, is expressed as

$$
\begin{aligned}
\overline{P s}_{i}= & \frac{m ! \alpha \Gamma(n-i+3 / 2) \beta^{i-n-1} i^{1-i}}{2 \sqrt{\pi}(i-1) !(m-i) !(n-i+1) ! \bar{\gamma}_{1}^{n-i+1}} \\
& +o\left(\bar{\gamma}_{1}^{-(n-i+1)}\right)
\end{aligned}
$$

The asymptotic ASEP at the 1st decoding layer is obtained as

$$
\overline{P s}_{1}=\frac{\alpha \Gamma(m n+1 / 2)}{2 \sqrt{\pi}(n !)^{m}\left(\beta \bar{\gamma}_{1}\right)^{m n}}+o\left(\bar{\gamma}_{1}^{-m n}\right) .
$$

\section{B. The error efficient approach}

Adopting the above methodology, the corresponding analysis for the error efficient approach is pursued as follows.

Proposition 3: The asymptotic CDF at the $i$ th decoding layer (i.e., the asymptotic outage probability), when $2 \leq i \leq m$, is expressed as

$$
\begin{aligned}
F_{\mathcal{X}_{i}}\left(\gamma_{\mathrm{th}}\right)= & \frac{m !(m-i+1)^{1-i}\left(\frac{\gamma_{\mathrm{th}}}{\bar{\gamma}_{1}}\right)^{n-i+1}}{(i-1) !(m-i) !(n-i+1) !} \\
& +o\left(\bar{\gamma}_{1}^{-(n-i+1)}\right) .
\end{aligned}
$$

The corresponding asymptotic CDF at the 1st decoding layer is equal to

$$
F_{\mathcal{X}_{1}}\left(\gamma_{\mathrm{th}}\right)=\frac{m}{n !}\left(\frac{\gamma_{\mathrm{th}}}{\bar{\gamma}_{1}}\right)^{n}+o\left(\bar{\gamma}_{1}^{-n}\right)
$$

Proposition 4: The asymptotic ASEP at the $i$ th decoding layer, when $2 \leq i \leq m$, is expressed as

$$
\begin{aligned}
\overline{P s}_{i}= & \frac{m ! \alpha \Gamma(n-i+3 / 2) \beta^{i-n-1}(m-i+1)^{1-i}}{2 \sqrt{\pi}(i-1) !(m-i) !(n-i+1) ! \bar{\gamma}_{1}^{n-i+1}} \\
& +o\left(\bar{\gamma}_{1}^{-(n-i+1)}\right) .
\end{aligned}
$$

The asymptotic ASEP at the 1st decoding layer is given by

$$
\overline{P s}_{1}=\frac{\alpha m \Gamma(m n+1 / 2)}{2 \sqrt{\pi} n !\left(\beta \bar{\gamma}_{1}\right)^{n}}+o\left(\bar{\gamma}_{1}^{-n}\right) .
$$

Collecting all the asymptotic results, we summarize the following insightful observations.

Remark 6: The diversity gain of the $i$ th decoding layer, when $i \geq 2$, is identical for both ordering approaches. Note that such an observation is not new and is consistent with some previous studies (e.g., [31], [46] and references therein). Specifically, it holds that $\mathcal{P}_{d_{i}}^{\text {(rate efficient) }}=\mathcal{S}_{d_{i}}^{\text {(rate efficient) }}=\mathcal{P}_{d_{i}}^{\text {(error efficient) }}=$ $\mathcal{S}_{d_{i}}^{\text {(error efficient) }}=n-i+1$. The difference lies in the 1 st layer (or the last SIC stage) where it holds that $\mathcal{P}_{d_{1}}^{\text {(rate efficient) }}=$ $\mathcal{S}_{d_{1}}^{\text {(rate efficient) }}=m n$ while $\mathcal{P}_{d_{1}}^{\text {(error efficient) }}=\mathcal{S}_{d_{1}}^{\text {(error efficient })}=n$.

This implies that the last SIC stage of the rate efficient approach delivers a considerably higher diversity order with respect to the last stage of the error efficient one.

Remark 7: The outage coding gains ${ }^{2}$ of the considered ordering approaches can be directly obtained as

$$
\mathcal{P}_{c_{i}}^{\text {(rate efficient })}=\left\{\begin{array}{cc}
\frac{1}{\gamma_{\mathrm{th}}}\left(\frac{((i-1) !)^{-1} m ! i^{1-i}}{(m-i) !(n-i+1) !}\right)^{-\frac{1}{n-i+1}}, & i \geq 2 \\
\frac{1}{\gamma_{\mathrm{th}}}\left(\frac{1}{(n !)^{m}}\right)^{-\frac{1}{m n}}, & i=1
\end{array}\right.
$$

\footnotetext{
${ }^{2}$ The ASEP coding gains can be straightforwardly derived in the same fashion as the outage coding gains.
} 


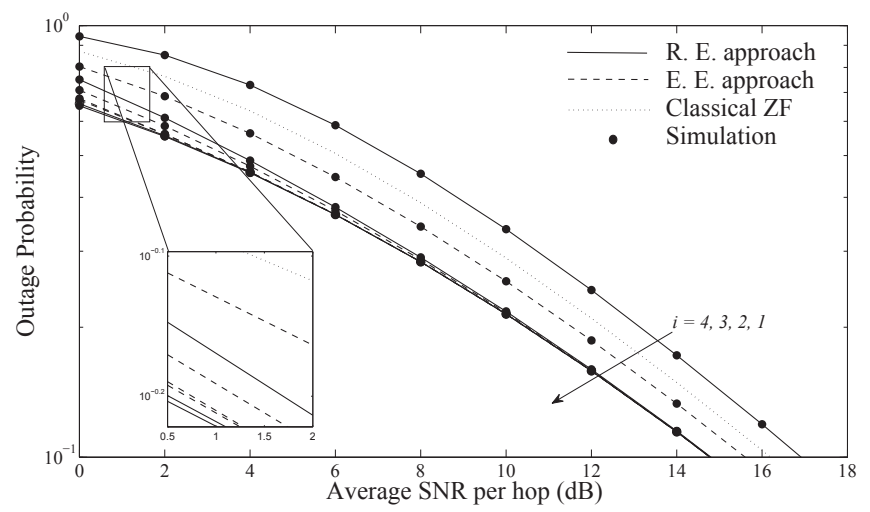

Fig. 2. Outage probability vs. average system SNR in strong atmospheric turbulence conditions, when $m=4, n=4, \gamma_{\mathrm{th}}=0 \mathrm{~dB}, \xi=6, \mathcal{A}=2.1$ and $\mathcal{B}=3.5$. The top-end curves correspond to the fourth decoding layer (or the first SIC stage) when $i=4$, the next ones to the third decoding layer (or the second SIC stage) when $i=3$ and so on.

and

$$
\begin{aligned}
& \mathcal{P}_{c_{i}}^{\text {(error efficient })}= \\
& \frac{1}{\gamma_{\text {th }}}\left(\frac{m !(m-i+1)^{1-i}}{(i-1) !(m-i) !(n-i+1) !}\right)^{-\frac{1}{n-i+1}} \quad, 1 \leq i \leq m .
\end{aligned}
$$

By closely observing the above asymptotic expressions, it is obvious that there is a performance gain for a reduced number of simultaneously transmitting source nodes (i.e., low $m$ values) and/or when the number of antennas at the relay is much higher than the number of source nodes (i.e., when $n>>m$ ). Interestingly, this scenario corresponds to massive (or very large-scale) MIMO systems, which are emerging at the forefront of wireless communications research [47]. Thus, assuming a massive MIMO-enabled relay node with a large antenna array used for reception (i.e., when $n \rightarrow \infty$ ), it is meaningful to investigate the performance gain improvement produced by the precise ordering. To this end, we introduce the relative performance coding gain (namely, RPCG), which is defined as

$\mathrm{RPCG} \triangleq \frac{\mathcal{P}_{c_{i}}^{\text {(rate efficient })}}{\mathcal{P}_{c_{i}}^{\text {(error efficient })}}=\left(\frac{i}{m-i+1}\right)^{\frac{i-1}{n-i+1}}, \quad 1<i \leq m$.

It is straightforward to show that $\lim _{n \rightarrow \infty} \mathrm{RPCG}=1$, which indicates no performance gain improvement between the two different ordering approaches, for asymptotically high $n$ values. The latter observation can be easily interpreted due to the flattening (in terms of channel fading) of all decoding layers, since they become mutually orthogonal when $n \rightarrow \infty$ [47, Eq. (13)].

\section{NumERICAL RESUlts AND Discussion}

In this section, analytical results are presented and compared with Monte-Carlo (MC) simulations. Each MC run was conducted over $10^{6} \mathrm{RV}$ trials. Symmetric average SNR per hop is assumed (i.e., $\bar{\gamma}_{1}=\bar{\gamma}_{2}$ ) for ease of tractability and without loss of generality. There is a good match between all the analytical and the respective simulation results and, hence, the accuracy of the proposed approach is verified. For

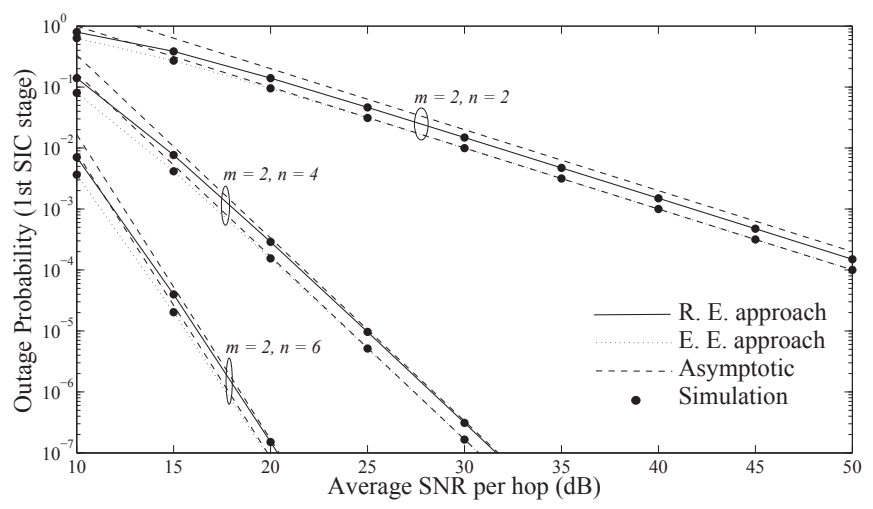

Fig. 3. Outage probability of the 1st SIC stage vs. average system SNR for various system setups in case of non-pointing errors (i.e., when $\xi \rightarrow \infty$ ), $\gamma_{\text {th }}=10 \mathrm{~dB}, \mathcal{A}=9.7$ and $\mathcal{B}=8.2$.

notational simplicity, in this section, we use the abbreviations R. E. and E. E. to define the rate efficient and the error efficient approach, respectively.

In order to better emphasize the impact of the ordered SIC at the relay, the proposed V-BLAST relaying scheme is compared against the conventional ZF detection approach [48]. Note that when the ZF reception is applied, all the transmitted signals are simultaneously detected at the relay without carrying out any kind of successive signal decoding. It is well-known that in the case of $\mathrm{ZF}$, the SNR of each transmitted signal (i.e., the $i$ th unordered source-to-relay SNR) can be expressed as [48] $\gamma_{1}^{(i)}=\bar{\gamma}_{1} /\left(\left(\mathbf{G}^{H} \mathbf{G}\right)^{-1}\right)_{i i}$. Also, the PDF of $\gamma_{1}^{(i)}$ follows the Erlang distribution with shape parameter $n-m+1$ and scale parameter $\bar{\gamma}_{1}$ [49]. Thus, based on (D.1), the CDF (i.e., the outage probability) of $\gamma_{e 2 e}$, identical for all the source nodes (due to the $\mathrm{ZF}$ principle and the i.i.d. fading channel assumption), is expressed as

$$
\begin{aligned}
F_{\gamma_{e 2 e}}^{(Z F)}(x) & =1-\frac{\xi^{2}}{\Gamma(\mathcal{A}) \Gamma(\mathcal{B})} G_{2,4}^{4,0}\left[\frac{\mathcal{A B} \sqrt{x}}{\sqrt{\bar{\gamma}_{2}}} \mid \begin{array}{c}
\xi^{2}+1,1 \\
0, \xi^{2}, \mathcal{A}, \mathcal{B}
\end{array}\right] \\
& \times \exp \left(-\frac{x}{\bar{\gamma}_{1}}\right) \sum_{k=0}^{n-m} \frac{\left(x / \bar{\gamma}_{1}\right)^{k}}{k !}
\end{aligned}
$$

It is obvious from Fig. 2 that E. E. always outperforms the conventional ZF, while only the 1 st SIC stage of R. E. is slightly inferior than ZF. Based on the proposed sorted QR decomposition, which is utilized prior to only the first SIC stage, a significant performance improvement (especially at the last SIC stages) is observed, as compared with ZF. As an indicative example, when the outage probability is $10^{-1}$, there is a performance gain of E. E. over ZF, which is in the order of $0.5 \mathrm{~dB}$ or $1.5 \mathrm{~dB}$, for the first or the subsequent SIC stages, respectively. As expected, the two different ordering strategies result to the superiority of E. E. against R. E. at the primary SIC stages and vice versa. Also, it is seen that the outage probability of the 1st SIC stage (for both approaches) serves as a lower system performance bound. This is due to the fact that all subsequent stages are detected/decoded with the presence of less interference and, thus, an increased performance gain.

In Fig. 3, this lower performance bound, i.e., only the outage probability of the 1st SIC stage (or the $m$ th decoding layer), is presented for several system configurations. The performance 


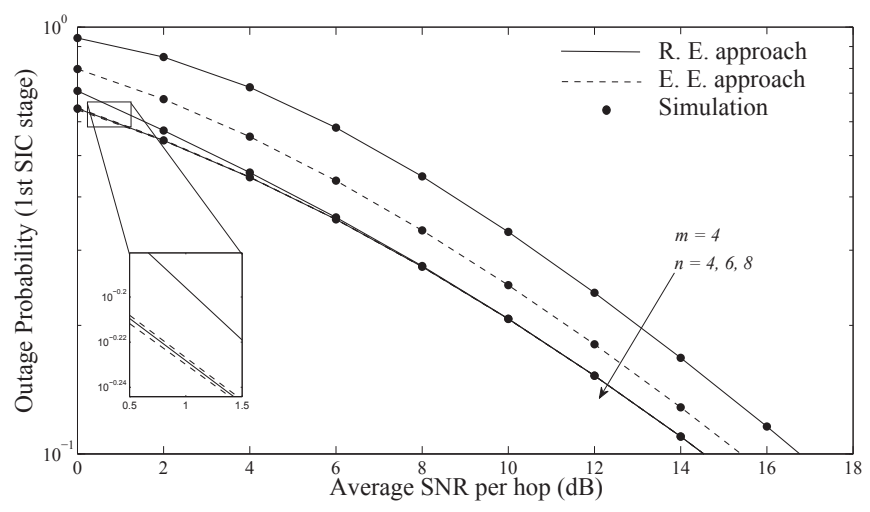

Fig. 4. Outage probability of the 1st SIC stage vs. average system SNR when $\xi \rightarrow \infty, m=4, \mathcal{A}=2.1$ and $\mathcal{B}=3.5$.

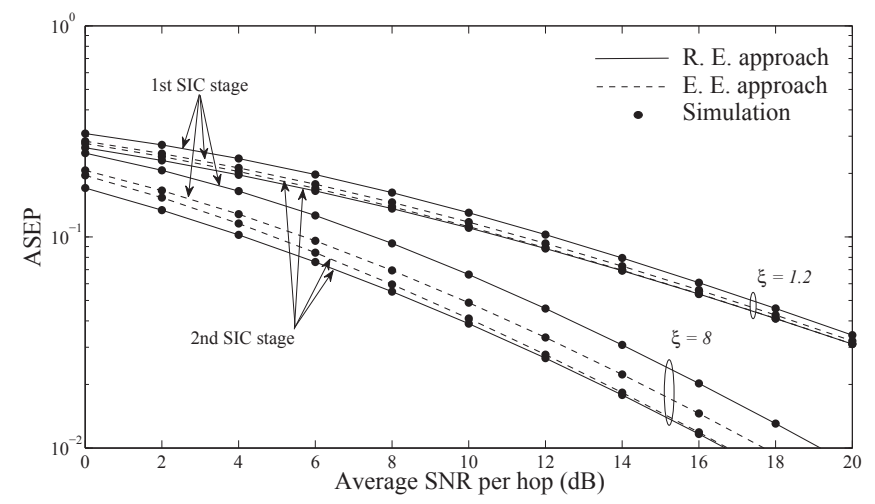

Fig. 5. ASEP vs. average system SNR for a BPSK modulation scheme, when $m=n=2, \mathcal{A}=4.4$ and $\mathcal{B}=2.6$.

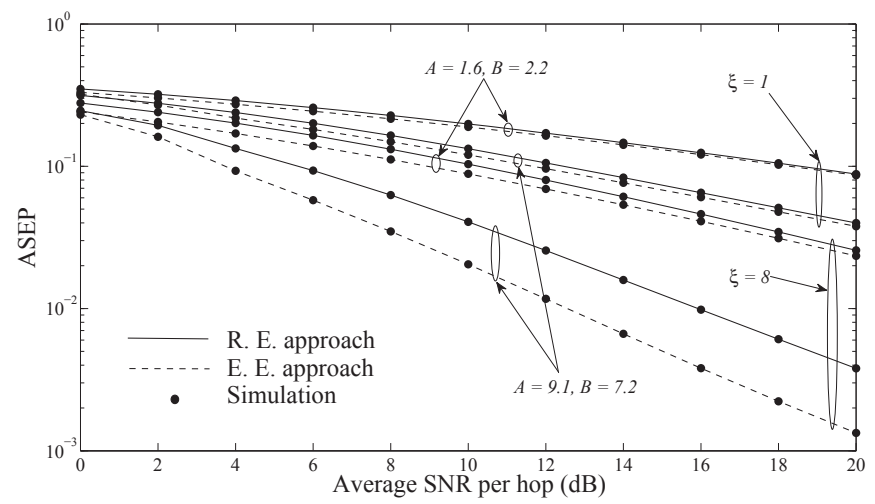

Fig. 6. ASEP vs. average system SNR for a BPSK modulation scheme, when $m=n=2$.

gain increases for a higher number of antennas at the relay. This agrees with the previously derived outage expressions and more emphatically with the corresponding asymptotic ones, e.g., see (29) and (33). Figure 4 indicates that the impact of the appropriate ordering on the system performance is more crucial when the number of receive antennas at the relay is similar to the number of simultaneously transmitting source nodes. Specifically, E. E. significantly outperforms R. E. when $m=n=4$, whereas such a performance gain becomes marginal as $n$ grows with a fixed number of source nodes (e.g., when $m=4$ and $n=8$ ). Finally, the influence of the pointing error displacement is depicted in Fig. 5, where the
ASEP performance loss is obvious for intense pointing errors in both SIC stages and/or ordering approaches. In addition, the fluctuation of the pointing error displacements seems to influence more drastically the end-to-end error performance than the heavy atmospheric turbulence conditions, especially for moderately medium/high SNRs (see Fig. 6).

\section{CONCLUSIONS}

We investigated a DF dual-hop relaying system over mixed RF/FSO links, which deploys the ordered ZF-SIC (V-BLAST) detection at the relay station. Analytical expressions for the outage probability and ASEP of the end-to-end link were derived in terms of finite sums of Meijer's- $G$ functions. Closedform asymptotic end-to-end outage and ASEP expressions were also presented. Capitalizing on the derived results, some useful insights were revealed. Particularly, the superiority of the proposed approach against the conventional ZF scheme was indicated with only a slight additional computational burden due to the initial symbol sorting. The diversity and coding gain for the end-to-end transmission of each source node were also provided. Moreover, it was shown that the ordering strategy offers marginal performance improvement when the number of receive antennas at the relay is much higher than the number of simultaneously transmitting source nodes. In addition, we demonstrated that the pointing error displacement is a more critical performance degradation factor than the turbulence-induced fading.

\section{APPENDIX A}

DERIVATION OF (8)

The (unconditional) PDF of the SNR at the $i$ th decoding layer, when $2 \leq i \leq m$, can be expressed as

$$
f_{\mathcal{X}_{i}}(x)=\int_{x}^{\infty} f_{\mathcal{X}_{i} \mid \mathcal{Y}_{i}}(x \mid y) f_{\mathcal{Y}_{i}}(y) d y .
$$

By applying (4) and (6) into (A.1), while utilizing the binomial expansion [37, Eq. (1.111)], we have that

$$
\begin{aligned}
& f_{\mathcal{X}_{i}}(x)= \\
& \sum_{j=0}^{i-2} \sum_{l=0}^{m-i} \frac{(-1)^{j+l}\left(\begin{array}{c}
i-2 \\
j
\end{array}\right)\left(\begin{array}{c}
m-i \\
l
\end{array}\right) x^{n+j-i}}{\bar{\gamma}_{1}^{n} B(n-i+1, i-1) B(m-i+1, i)(n-1) !} \\
& \int_{x}^{\infty} y^{i-j-2} \exp \left(-\frac{(i+l)}{\bar{\gamma}_{1}} y\right)\left(\sum_{k=0}^{n-1} \frac{\left(y / \bar{\gamma}_{1}\right)^{k}}{k !}\right)^{i+l-1} d y
\end{aligned}
$$

To evaluate the multinomial power within the above integral, we reproduce the following useful formula [50, Eq. (9)]

$$
\begin{aligned}
& \left(\sum_{k=0}^{n-1} \frac{\left(y / \bar{\gamma}_{1}\right)^{k}}{k !}\right)^{i+l-1}=\sum_{p_{1}=0}^{i+l-1} \sum_{p_{2}=0}^{p_{1}} \cdots \sum_{p_{n-1}=0}^{p_{n-2}}\left(y / \bar{\gamma}_{1}\right)^{\phi} \\
& \times \frac{(i+l-1) !}{p_{n-1} !} \prod_{t=1}^{n-1}\left[\frac{1}{\left(p_{t-1}-p_{t}\right) !(t !)^{p_{t}-p_{t+1}}}\right] .
\end{aligned}
$$

Recall that $p_{0}, p_{n}$ and $\phi$ have already been defined in (9). Hence, plugging (A.3) into (A.2), utilizing [37, Eq. (3.351.2)] and after some straightforward manipulations, the closed-form PDF at the $i$ th layer of the rate efficient approach is obtained in (8). 


\section{APPENDIX B}

DERIVATION OF (11)

Keeping in mind Remark 4 and based on (4), we have that

$$
\begin{aligned}
f_{\mathcal{X}_{1}}(x) & =\frac{m x^{n-1} \exp \left(-\frac{x}{\bar{\gamma}_{1}}\right)}{\bar{\gamma}_{1}^{n}(n-1) !} \\
& \times\left(1-\exp \left(-\frac{x}{\bar{\gamma}_{1}}\right) \sum_{k=0}^{n-1} \frac{\left(x / \bar{\gamma}_{1}\right)^{k}}{k !}\right)^{m-1} .
\end{aligned}
$$

Utilizing the binomial expansion [37, Eq. (1.111)] and the multinomial identity as given in (A.3), the corresponding closed-form PDF can be derived.

\section{APPENDIX C}

\section{DERIVATION OF (14)}

Following the same methodology as presented in Appendix A, but using (3) instead of (4), the derivations of $\Delta_{1}$ and $\Delta_{2}$ can be easily obtained after some straightforward manipulations. Also, keeping in mind Remark 4 and based on (3), we have that

$$
\begin{aligned}
& f_{\mathcal{X}_{1}}(x)= \\
& \frac{m x^{n-1} \exp \left(-\frac{x}{\bar{\gamma}_{1}}\right)}{\bar{\gamma}_{1}^{n}(n-1) !}\left(\exp \left(-\frac{x}{\bar{\gamma}_{1}}\right) \sum_{k=0}^{n-1} \frac{\left(x / \bar{\gamma}_{1}\right)^{k}}{k !}\right)^{m-1} .
\end{aligned}
$$

Utilizing the multinomial identity as given in (A.3), the corresponding closed-form PDF/CDF of the SNR at the 1st decoding layer can be derived following the same methodology as for the rate efficient approach.

\section{APPENDIX D}

DERIVATION OF (16) AND (17)

Based on (1), we have that

$$
\begin{aligned}
F_{\gamma_{e 2 e}}^{(i)}(x) & \triangleq 1-\operatorname{Pr}\left\{\gamma_{1}^{(i)}=\mathcal{X}_{i}>x\right\} \operatorname{Pr}\left\{\gamma_{2}>x\right\} \\
& =1-\underbrace{\int_{x}^{\infty} f_{\gamma_{1}^{(i)}}(\omega) d \omega}_{\mathcal{I}_{1}} \underbrace{\int_{x}^{\infty} f_{\gamma_{2}}(\omega) d \omega}_{\mathcal{I}_{2}},
\end{aligned}
$$

where $\operatorname{Pr}\{$.$\} denotes probability and F_{\gamma_{e 2 e}}^{(i)}($.$) is the \mathrm{CDF}$ of the end-to-end SNR between the $i$ th source node and the destination.

Using (8) and (11) while invoking [37, Eq. (3.351.2)], yields

$$
\begin{aligned}
\mathcal{I}_{1} & =\Xi(n, m, i) \sum_{r=0}^{i+\phi-j-2} \sum_{k=0}^{r+n+j-i} \frac{(i+\phi-j-2) !}{r ! k !\left(\frac{\Delta_{2}}{\bar{\gamma}_{1}}\right)^{\phi+n-k}} \\
& \times(r+n+j-i) ! x^{k} \exp \left(-\frac{\Delta_{2}}{\bar{\gamma}_{1}} x\right), \quad 2 \leq i \leq m,
\end{aligned}
$$

and

$$
\mathcal{I}_{1}=\Psi(n, m) \sum_{k=0}^{n+\phi-1} \frac{(n+\phi-1) ! x^{k} \exp \left(-\frac{j+1}{\bar{\gamma}_{1}} x\right)}{k !\left(\frac{j+1}{\bar{\gamma}_{1}}\right)^{n+\phi-k}}, i=1 .
$$

Next, to evaluate $\mathcal{I}_{2}$, we have that

$$
\begin{aligned}
\mathcal{I}_{2} & =\int_{x}^{\infty} \frac{\xi^{2}}{2 \omega \Gamma(\mathcal{A}) \Gamma(\mathcal{B})} G_{1,3}^{3,0}\left[\mathcal{A B} \sqrt{\frac{\omega}{\bar{\gamma}_{2}}} \mid \begin{array}{c}
\xi^{2}+1 \\
\xi^{2}, \mathcal{A}, \mathcal{B}
\end{array}\right] d \omega \\
& \stackrel{(a)}{=} \int_{0}^{1 / \sqrt{x}} \frac{\xi^{2}}{\omega \Gamma(\mathcal{A}) \Gamma(\mathcal{B})} G_{1,3}^{3,0}\left[\frac{\mathcal{A B}}{\sqrt{\bar{\gamma}_{2}} \omega} \mid \begin{array}{c}
\xi^{2}+1 \\
\xi^{2}, \mathcal{A}, \mathcal{B}
\end{array}\right] d \omega \\
& \stackrel{(b)}{=} \frac{\xi^{2}}{\Gamma(\mathcal{A}) \Gamma(\mathcal{B})} G_{2,4}^{4,0}\left[\frac{\mathcal{A B} \sqrt{x}}{\sqrt{\bar{\gamma}_{2}}} \mid \begin{array}{c}
\xi^{2}+1,1 \\
0, \xi^{2}, \mathcal{A}, \mathcal{B}
\end{array}\right],
\end{aligned}
$$

where $(a)$ is obtained by applying a change of variables (first $\sqrt{\omega} \rightarrow \omega$ and then $\omega \rightarrow 1 / \omega)$, while $(b)$ is derived with the aid of [37, Eq. (9.31.2)] and [41, Eq. (26)]. Hence, plugging (D.2) or (D.3) and (D.4) into (D.1), while setting $x=\gamma_{\mathrm{th}}$, gives (16) or (17), respectively.

\section{APPENDIX E}

DERIVATION OF (29) AND (30)

In the asymptotically high average SNR regime, only the first summation term of (10) should be taken into account. All the remaining terms approach zero as $\bar{\gamma}_{1} \rightarrow \infty$. Hence, based on (28), keeping only the first-order term in (10) while recognizing that $\gamma(\alpha, x) \approx x^{\alpha} / \alpha$ as $x \rightarrow 0$ [37, Eq. (8.354.1)], (29) can be obtained after some simple manipulations. Moreover, recalling Remark 4 and using (4), it can be seen that the PDF of the 1st layer may be alternatively expressed as

$$
\begin{aligned}
f_{\mathcal{X}_{1}}(x) & =\frac{m x^{n-1} \exp \left(-\frac{x}{\bar{\gamma}_{1}}\right)}{\bar{\gamma}_{1}^{n}(n-1) !} \\
& \times\left(\exp \left(-\frac{x}{\bar{\gamma}_{1}}\right) \sum_{k=n}^{\infty} \frac{\left(x / \bar{\gamma}_{1}\right)^{k}}{k !}\right)^{m-1} .
\end{aligned}
$$

Thus, retaining only the first order term in (E.1) and evaluating the corresponding $\mathrm{CDF}$, we arrive at (30).

\section{REFERENCES}

[1] R. Pabst et al., "Relay-based deployment concepts for wireless and mobile broadband radio," IEEE Commun. Mag., vol. 42, no. 9, pp. 80-89, Sep. 2004.

[2] S. S. Ikki, S. Al-Dharrab, and M. Uysal, "Error probability of DF relaying with pilot-assisted channel estimation over time-varying fading channels," IEEE Trans. Veh. Technol., vol. 61, no. 1, pp. 393-397, Jan. 2012.

[3] R. Wang, M. Tao, and Y. Huang, "Linear precoding designs for amplifyand-forward multiuser two-way relay systems," IEEE Trans. Wireless Commun., vol. 11, no. 12, pp. 4457-4469, Oct. 2012.

[4] K. T. Hemachandra and N. C. Beaulieu, "Outage analysis of opportunistic scheduling in dual-hop multiuser relay networks in the presence of interference," IEEE Trans. Commun., vol. 61, no. 5, pp. 1786-1796, May 2013.

[5] Z. K. M. Ho and E. A. Jorswieck, "Instantaneous relaying: optimal strategies and interference neutralization," IEEE Trans. Signal Process., vol. 60, no. 12, pp. 6655-6668, Nov. 2012.

[6] M. Safari and M. Uysal, "Relay-assisted free-space optical communication," IEEE Trans. Wireless Commun., vol. 7, no. 12, pp. 5441-5449, Dec. 2008 .

[7] N. D. Chatzidiamantis, H. G. Sandalidis, G. K. Karagiannidis, and M. Matthaiou, "Inverse Gaussian modeling of turbulence-induced fading in free-space optical systems," J. Lightw. Technol., vol. 29, no. 10, pp. 15901596, May 2011.

[8] M. Uysal, J. Li, and M. Yu, "Error rate performance analysis of coded free-space optical links over gamma-gamma atmospheric turbulence channels," IEEE Trans. Wireless Commun., vol. 5, no. 6, pp. 1229-1233, June 2006.

[9] N. D. Chatzidiamantis, A. S. Lioumpas, G. K. Karagiannidis, and S. Arnon, "Adaptive subcarrier PSK intensity modulation in free space optical systems," IEEE Trans. Commun., vol. 59, no. 5, pp. 1368-1377, May 2011. 
[10] A. G. Zambrana, C. C. Vazquez, and B. C. Vazquez, "Outage performance of MIMO FSO links over strong turbulence and misalignment fading channels," Opt. Express, vol. 19, no. 14, pp. 13480-13496, June 2011.

[11] A. A. Farid and S. Hranilovic, "Outage capacity optimization for freespace optical links with pointing errors," J. Lightw. Technol., vol. 25, no. 7, pp. 1702-1710, July 2007.

[12] Z. Ghassemlooy et al., "Performance analysis of Ethernet/fast-Ethernet free space optical communications in a controlled weak turbulence condition," J. Lightw. Technol., vol. 30, no. 13, pp. 2188-2194, July 2012.

[13] G. Parca et al., "Optical wireless transmission at 1.6-Tbit/s $(16 \times 100$ Gbit/s) for next-generation convergent urban infrastructures," Opt. Eng., vol. 52, no. 11, Nov. 2013.

[14] S. Zvanovec et al., "Route diversity analyses for free-space optical wireless links within turbulent scenarios," Opt. Express, vol. 21, no. 6, pp. 7641-7650, Mar. 2013.

[15] E. Lee, J. Park, D. Han, and G. Yoon, "Performance analysis of the asymmetric dual-hop relay transmission with mixed RF/FSO links," IEEE Photonics Technol. Lett., vol. 23, no. 21, pp. 1642-1644, Nov. 2011.

[16] I. S. Ansari, F. Yilmaz, and M.-S. Alouini, "Impact of pointing errors on the performance of mixed RF/FSO dual-hop transmission systems," IEEE Wireless Commun. Lett., vol. 2, no. 3, pp. 351-354, June 2013.

[17] J. Liao et al., "Packaging of PIN photodiode on patch antenna for a dualmode indoor RF/FSO receiver," IEEE Trans. Compon. Packag. Manuf. Technol., vol. 1, no. 3, pp. 335-343, Mar. 2011.

[18] W. Zhang, S. Hranilovic, and C. Shi, "Soft-switching hybrid FSO/RF links using short-length Raptor codes: design and implementation," IEEE J. Sel. Areas Commun., vol. 27, no. 9, pp. 1698-1708, Dec. 2009.

[19] B. He and R. Schober, "Bit-interleaved coded modulation for hybrid RF/FSO systems," IEEE Trans. Commun., vol. 57, no. 12, pp. 37533763, Dec. 2009.

[20] Z. Wang and W. Chen, "Relay beamforming design with SIC detection for MIMO multi-relay networks with imperfect CSI," IEEE Trans. Veh. Technol., vol. 62, no. 8, pp. 3774-3785, Oct. 2013.

[21] N. I. Miridakis, "Performance analysis of V-BLAST reception under multiuser decode-and-forward cooperation," IET Commun., to be published, 2014.

[22] J. Cao, Z. Zhong, and F. Wang, "Regenerative multi-way relaying: relay precoding and ordered MMSE-SIC receiver," in Proc. 2012 IEEE Veh. Techn. Conf. - Spring.

[23] E. Chiu, V. K. N. Lau, Z. Shunqing, and B. S. M. Mok, "Precoder design for multi-antenna partial decode-and-forward (pdf) cooperative systems with statistical CSIT and MMSE-SIC receivers," IEEE Trans. Wireless Commun., vol. 11, no. 4, pp. 1343-1349, Apr. 2012.

[24] Y. Kim, W. Choi, B. Jung, and A. Nosratinia, "A dynamic paradigm for spectrally efficient half-duplex multi-antenna relaying," IEEE Trans. Wireless Commun., vol. 12, no. 9, pp. 4680-4691, Sept. 2013.

[25] R. P. Sirigina, A. S. Madhukumar, and L. Qian, "Performance analysis of Z-channel with relay under Rayleigh fading and discrete constellations," in Proc. 2012 IEEE Wireless Commun. Netw. Conf., pp. 92-96.

[26] N. I. Miridakis and D. D. Vergados, "Performance analysis of the ordered V-BLAST approach over Nakagami- $m$ fading channels," IEEE Wireless Commun. Lett., vol. 2, no. 1, pp. 18-21, Feb. 2013.

[27] —, "Efficient detection-switching based on successive interference cancellation for multiple-input multiple-output orthogonal frequency division multiplexing systems," IET Signal Process., vol. 7, no. 5, pp. 436-443, June 2013.

[28] H. Dai, A. F. Molisch, and H. V. Poor, "Downlink capacity of interference-limited MIMO systems with joint detection," IEEE Trans. Wireless Commun., vol. 3, no. 2, pp. 442-453, Mar. 2004.

[29] J. N. Laneman, D. N. C. Tse, and G. W. Wornell, "Cooperative diversity in wireless networks: efficient protocols and outage behavior," IEEE Trans. Inf. Theory., vol. 50, no. 12, pp. 3062-3080, Dec. 2004.

[30] S. Ozyurt and M. Torlak, "An exact outage analysis of zero-forcing V-BLAST with greedy ordering," in Proc. 2011 IEEE Global Commun. Conf., pp. 1399-1403.

[31] Y. Jiang and M. K. Varanasi, "Spatial multiplexing architectures with jointly designed rate-tailoring and ordered BLAST decoding-part I: diversity-multiplexing tradeoff analysis," IEEE Trans. Wireless Commun., vol. 7, no. 8, pp. 3252-3261, Aug. 2008.

[32] Y. Jiang, M. K. Varanasi, and J. Li, "Performance analysis of ZF and MMSE equalizers for MIMO systems: an in-depth study of the high SNR regime," IEEE Trans. Inf. Theory, vol. 57, no. 4, pp. 2008-2026, Apr. 2011.

[33] S. Loyka and F. Gagnon, "Performance analysis of the V-BLAST algorithm: an analytical approach," IEEE Trans. Wireless Commun., vol. 3 , no. 4, pp. 1326-1337, July 2004.
[34] J. Choi, "A bi-directional zero-forcing BLAST receiver," IEEE Trans. Signal Process., vol. 52, no. 9, pp. 2670-2673, Sept. 2004.

[35] C. Ling, W. H. Mow, and L. Gan, "Dual-lattice ordering and partial lattice reduction for SIC-based MIMO detection," IEEE J. Sel. Topics Signal Process., vol. 3, no. 6, pp. 975-985, Jan. 2010.

[36] H.-C. Yang and M.-S. Alouini, Order Statistics in Wireless Communications. Cambridge University Press, 2011.

[37] I. S. Gradshteyn and I. M. Ryzhik, Table of Integrals, Series and Products, 7th ed. Academic Press, 2007.

[38] R. J. Muirhead, Aspects of Multivariate Statistical Theory, Wiley Series in Probability and Statistics, 1982.

[39] H. G. Sandalidis, T. A. Tsiftsis, and G. K. Karagiannidis, "Optical wireless communications with heterodyne detection over turbulence channels with pointing errors," J. Lightw. Technol., vol. 27, no. 20, pp. 4440-4445, Oct. 2009.

[40] G. J. Foschini, G. D. Golden, R. A. Valenzuela, and P. W. Wolniansky, "Simplified processing for high spectral efficiency wireless communication employing multi-element arrays," IEEE J. Sel. Areas Commun., vol. 17, no. 11, pp. 1841-1852, Nov. 1999.

[41] V. S. Adamchik and O. I. Marichev, "The algorithm for calculating integrals of hypergeometric type functions and its realization in REDUCE system," in Proc. 1990 Inter. Conf./Symp. Symbolic Algebraic Computation, pp. 212-224.

[42] W. O. Popoola and Z. Ghassemlooy, "BPSK subcarrier intensity modulated free-space optical communications in atmospheric turbulence," $J$. Lightw. Technol., vol. 27, no. 8, pp. 967-973, Apr. 2009.

[43] M. R. McKay, A. J. Grant, and I. B. Collings, "Performance analysis of MIMO-MRC in double-correlated Rayleigh environments," IEEE Trans. Commun., vol. 55, no. 3, pp. 497-507, Mar. 2007.

[44] A. P. Prudnikov, Y. A. Brychkov, and O. I. Marichev, Integral and Series. Vol. 3: More Special Functions. Gordon and Breach Science Publishers, 1986.

[45] Z. Wang and G. B. Giannakis, "A simple and general parameterization quantifying performance in fading channels," IEEE Trans. Commun., vol. 51, no. 8, pp. 1389-1398, Aug. 2003.

[46] S. Loyka and F. Gagnon, "On outage and error rate analysis of the ordered V-BLAST," IEEE Trans. Wireless Commun., vol. 7, no. 10, pp. 3679-3685, Oct. 2008.

[47] F. Rusek et al., "Scaling up MIMO: opportunities and challenges with very large arrays," IEEE Signal Process. Mag., vol. 30, no. 1, pp. 40-60, Jan. 2013.

[48] M. Matthaiou, C. Zhong, and T. Ratnarajah, "Novel generic bounds on the sum rate of MIMO ZF receivers," IEEE Trans. Signal Process., vol. 59, no. 9, pp. 4341-4353, Sept. 2011.

[49] D. Lee, "Performance analysis of ZF-precoded scheduling system for MU-MIMO with generalized selection criterion," IEEE Trans. Wireless Commun., vol. 12, no. 4, pp. 1812-1818, Apr. 2013.

[50] S. Choi and Y.-C. Ko, "Performance of selection MIMO systems with generalized selection criterion over Nakagami- $m$ fading channels," IEICE Trans. Commun., vol. E89-B, no. 12, pp. 3467-3470, Dec. 2006.

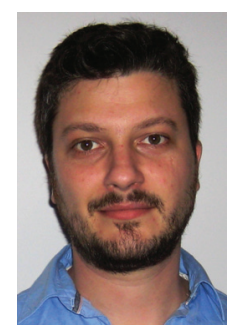

Nikolaos I. Miridakis was born in Athens, Greece. $\mathrm{He}$ received his M.Sc. and Ph.D. degrees in Networking and Data Communications from the Department of Information Systems, Kingston University, U.K. in 2008 and from the Department of Informatics, University of Piraeus, Greece in 2012, respectively. Since 2007 , he has been with the Department of Computer Engineering, Technological Education Institute of Piraeus, Greece where he is a Teaching Cooperator and Research Associate. Also, since 2012, he has been with the Department of Informatics, University of Piraeus, Greece where he is a Senior Research Associate. His research interests include wireless communications, and more specifically interference analysis and management in wireless communications, multicarrier communications, MIMO systems, statistical signal processing, diversity reception, fading channels, and cooperative communications.

Dr. Miridakis serves as a reviewer for several prestigious international journals and conferences. 


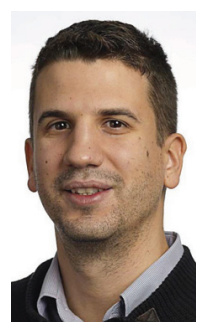

Michail Matthaiou (S'05-M'08-SM'13) was born in Thessaloniki, Greece in 1981. He obtained the Diploma degree (5 years) in Electrical and Computer Engineering from the Aristotle University of Thessaloniki, Greece in 2004. He then received the M.Sc. (with distinction) in Communication Systems and Signal Processing from the University of Bristol, U.K. and Ph.D. degrees from the University of Edinburgh, U.K. in 2005 and 2008, respectively. From September 2008 through May 2010, he was with the Institute for Circuit Theory and Signal Processing, Munich University of Technology (TUM), Germany working as a Postdoctoral Research Associate. He is currently a Senior Lecturer at Queen's University Belfast, U.K. and also holds an adjunct Assistant Professor position at Chalmers University of Technology, Sweden. His research interests span signal processing for wireless communications, massive MIMO, hardwareconstrained communications, and performance analysis of fading channels.

Dr. Matthaiou is the recipient of the 2011 IEEE ComSoc Young Researcher Award for the Europe, Middle East and Africa Region and a co-recipient of the 2006 IEEE Communications Chapter Project Prize for the best M.Sc. dissertation in the area of communications. He was an Exemplary Reviewer for IEEE COMMUNICATIONS LETTERS for 2010. He has been a member of Technical Program Committees for several IEEE conferences such as ICC, GLOBECOM, VTC etc. He currently serves as an Associate Editor for the IEEE TRANSACTIONS ON COMMUNICATIONS, IEEE COMMUNICATIONS LETTERS and was the Lead Guest Editor of the special issue on "Large-scale multiple antenna wireless systems" of the IEEE JOURNAL ON SELECTED AREAS IN COMMUNICATIONS. He is an associate member of the IEEE Signal Processing Society SPCOM and SAM technical committees.

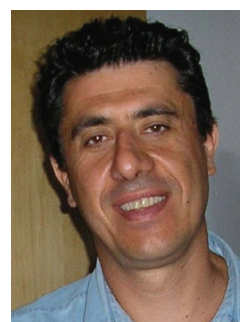

George K. Karagiannidis (SM'03-F'14) was born in Pithagorion, Samos Island, Greece. He received the University Diploma (5 years) and Ph.D degree, both in electrical and computer engineering from the University of Patras, in 1987 and 1999, respectively. From 2000 to 2004, he was a Senior Researcher at the Institute for Space Applications and Remote Sensing, National Observatory of Athens, Greece. In June 2004, he joined the faculty of Aristotle University of Thessaloniki, Greece where he is currently Professor and Director of Digital Telecommunications Systems and Networks Laboratory. His research interests are in the broad area of digital communications systems with emphasis on communications theory, energy efficient MIMO and cooperative communications, cognitive radio, smart grid and optical wireless communications. He is the author or coauthor of more than 240 technical papers published in scientific journals and presented at international conferences. He is also author of the Greek edition of a book on "Telecommunications Systems" and co-author of the book Advanced Wireless Communications Systems (Cambridge Publications, 2012). $\mathrm{He}$ is co-recipient of the Best Paper Award of the Wireless Communications Symposium (WCS) in the IEEE International Conference on Communications (ICC'07), Glasgow, U.K., June 2007.

Dr. Karagiannidis has been a member of Technical Program Committees for several IEEE conferences such as ICC, GLOBECOM, VTC, etc. In the past he was Editor for Fading Channels and Diversity of the IEEE TRANSACTIONS ON COMMUNiCATIONS, Senior Editor of IEEE COMMUNICATIONS LETTERS and Editor of the EURASIP Journal of Wireless Communications and Networks. He was Lead Guest Editor of the special issue on "Optical wireless communications" of the IEEE JOURNAL ON SELECTED AREAS IN COMmunications and Guest Editor of the special issue on "Large-scale multiple antenna wireless systems". Since January 2012, he is the Editor-in Chief of the IEEE COMMUNICATIONS LETTERS. 